\title{
Dinámicas Urbanas, Actividades Emergentes Políticas Públicas en la Región Metropolitana de Barcelona
}

Oriol Nel $\cdot \mathrm{lo}^{*}$

\begin{abstract}
$\mathrm{R}$ A região metropolitana de Barcelona, como tantas áreas metropolitanas da Europa encontra-se imersa num acelarado processo de mudança. São três as suas principais características: a extensão da área urbana sobre um âmbito cada vez maior, a dispersão da população e actividades sobre o espaço e a especialização funcional e social de cada área em função do conjunto metropolitano. Estas transformações apresentam, em conjunto, numerosas oportunidades, importantes desafios, em relação sobretudo à sustentabilidade ambiental, à eficiência funcional e à segregação social. As políticas urbanas aplicadas para fazer frente a estes desafios apontam, com as suas luzes e sombras para a defesa do modelo de cidade compacta, complexa e coesa. Como exemplo destas políticas analisam-se as actuações relativas à actividade turíslica, à nova indústria e ao equilíbrio social na cidade central da área: o município de Barcelona.
\end{abstract}

Palavras-chave: área metropolitana de Barcelona; dinâmicas de transformação urbana; políticas urbanas.

\section{Introducción}

Barcelona ha sido a lo largo de los dos últimos siglos una ciudad eminentemente industrial. Aquí se instaló - en 1832 - la primera industria de vapor de España, aquí se construyó - en 1848 - la primera línea de ferrocarril, aquí acabaría formándose el principal polo fabril, el puerto más activo, la más populosa concentración obrera. Tanto es así, que la imagen de la ciudad se relacionó durante muchos años a la actividad industrial y, también, a la conflictividad social que su desarrollo llevó aparejado: la "fábrica de España", la "ciudad de las bombas", la "rosa de fuego".

$\mathrm{Si}$, en los años selenta, hubiéramos podido explicar a un residente del Poblenou - el barrio más industrial de la ciudad más industrial, el "Manchester catalán", cuna del movimiento obrero y anarquista - que, apenas treinta años más tarde las grandes fábricas (Tilan, Motor Ibérica, la Maquinista Terrestre y Marítima,...) habrían desparecido completamente y en su lugar florecería un distrito turístico, residencial y cultural, difícilmente nos habría dado ningún crédito.

Pues bien, esto es exactamente lo que ha ocurrido. En el último cuarlo de siglo Barcelona ha conocido una transformación radical: la base económica, la estructura social, los hábitos de su población, la estructura física e incluso la imagen misma de la ciudad han conocido cambios acelerados y decisivos. Algunas de estas transformaciones han sido tan espectaculares que incluso han atraído atención internacional.

El objeto de este papel es precisamente aporLar algunos datos y reflexiones sobre la naturaleza de estos cambios, su relación con la aparición de nuevos sectores o clusters de actividad y las políticas públicas que en la ciudad se han aplicado. Así, el trabajo tratará de exponer, en otros tantos apartados, los cinco postulados siguientes:

a) Barcelona constituye hoy una realidad metropolitana sujeta a una intensa dinámica de transformación, a través de la cual la ciudad tiende

\footnotetext{
* Professor do Departament de Cieografia de la Universitat Autònoma de Barcelona. Deputado no Parlamento da Catalunha. Contacto: Onello@parlament-cat.es
} 
a integrar un espacio siempre más vasto, a ver dispersarse sus actividades y población, así como a especializar funcional y socialmente las distintas áreas que la integran.

b) Esta transformación, que se ha visto acompañada por un cambio estructural de la base económica y un aumento muy notable de los niveles de renta medios, ofrece notables oportunidades entre las que cabe destacar las que se derivan del salto de escala metropolitano y del mejor acceso a los servicios y la ocupación para el conjunto del área urbana.

c) Ahora bien, estas oportunidades se ven acompañadas también de retos importantes que pueden llegar a poner en riesgo la sostenibilidad ambiental, la eficiencia funcional y la cohesión social de la ciudad.

d) Ante estas oportunidades y riesgos, se ha tratado de desarrollar un conjunto de políticas tendentes a salvaguardar la competitividad, la funcionalidad y la calidad de vida en la ciudad central, a través de la defensa de un modelo de urbanización caracterizado por la densidad, la complejidad y la cohesión social.

e) El éxito de estas políticas - en cuya aplicación pueden encontrarse luces y sombras depende, en muy buena medida, de la capacidad de contribuir al desarrollo de actividades emergentes como el turismo y el ocio, las nuevas formas de producción y la creación cultural. Se trata de actividades con una tradición relativamente reciente en la ciudad central pero de rápido desarrollo, como muestran de los ejemplos de la evolución de los nuevos centros turísticos, la operación 22@ y la experiencia del Forum 2004.

\section{Las Dinámicas de Transformación Urbana}

Decíamos que el ámbito metropolitano de Barcelona conoce hoy un acelerado proceso de cambio. Tres son las tendencias características de esta transformación, las cuales son, como se verá, coincidentes, en buena parte, con las dinámicas de transformación de la mayoría de las grandes ciudades de la Península Ibérica y de Europa Occidental: a) Dispersión. La población y las actividades, después de un largo proceso caracterizado por una tendencia muy acentuada hacia la concentración, se dispersan ahora sobre el espacio metropolitano.

b) Extensión. Al tiempo que se dispersa sobre el territorio el área urbana tiende a expandirse progresivamente para integrar en el ámbito que puede ser considerado como metropolitano un territorio siempre más vasto.

c) Especialización. Esta ciudad que se dispersa y se expande tiende además a especializar funcional y socialmente cada una de las áreas que la integran en función del conjunto.

Veamos con un poco de detalle como actúan estas dinámicas. Pero antes de adentrarnos en su estudio, será conveniente un breve recordatorio de las magnitudes de las que estamos hablando.

\section{La Región Metropolitana de Barce- lona: magnitudes básicas}

Los datos básicos referentes a territorio, actividad económica y población de la Barcelona metropolitana son los siguientes:

- El ámbito metropolitano de Barcelona, en su definición administrativa, es un territorio de $3235 \mathrm{Km}^{2}$, lo cual viene a representar algo más que el 10\% del territorio de la región catalana.

- El PIB del ámbito metropolitano representa el $69 \%$ del total catalán y a un $13,4 \%$ del total español, con un montante total 14,4 billones de pesetas (el PIB catalán es de 20,9 BPTA y el español de 107,8 BPTA según las estimaciones disponibles para el año 2001). Asimismo hay que reseñar que la provincia de Barcelona absorbe el $25,6 \%$ de las importaciones españolas y genera el $22,3 \%$ de las exportaciones.

- La población total de la Barcelona metropolitana que había crecido de manera incesante y muy acentuada en los años sesenta y primeros setenta, se ha mantenido prácticamente estable desde 1981. En 1996, según los datos del último padrón disponible, alcanzaba los 4228047 habitantes. Esto representa el 69,4\% de la población catalana.

- Esta población se encuentra muy desigualmente repartida, de modo que en los escasos 100 $\mathrm{Km}^{2}$ del municipio de Barcelona se encuentran 1,5 millones de habitantes, en una treintena de 
municipios circumstantes (sobre una superficie de $378 \mathrm{Km}^{2}$ ), otros 1,3 millones, y, finalmente, en los $2759 \mathrm{Km}^{2}$ restantes del territorio metropolitano habitan otros 1,4 millones. Así, en un 1\% del territorio de Catalunya se encuentra una cuarta parte de la población, en un $2 \%$ cerca de la mitad y en un $10 \%$ cerca de los tres cuartos.

- Hay que hacer notar, finalmente, que la estructura urbana del área metropolitana es compleja y rica, al contar, junto a la ciudad central, con un conjunto de ciudades medias - de entre 30000 y 200000 habitantes - algunas de las cuales tienen una importante tradición industrial y comercial (como Mataró, Granollers, Sabadell, Terrassa, Vilanova o Vilafranca) y otras se están afirmando como centros residenciales y terciarios emergentes (como Sant Cugat, Mollet, Cerdanyola, Sitges o Calella).

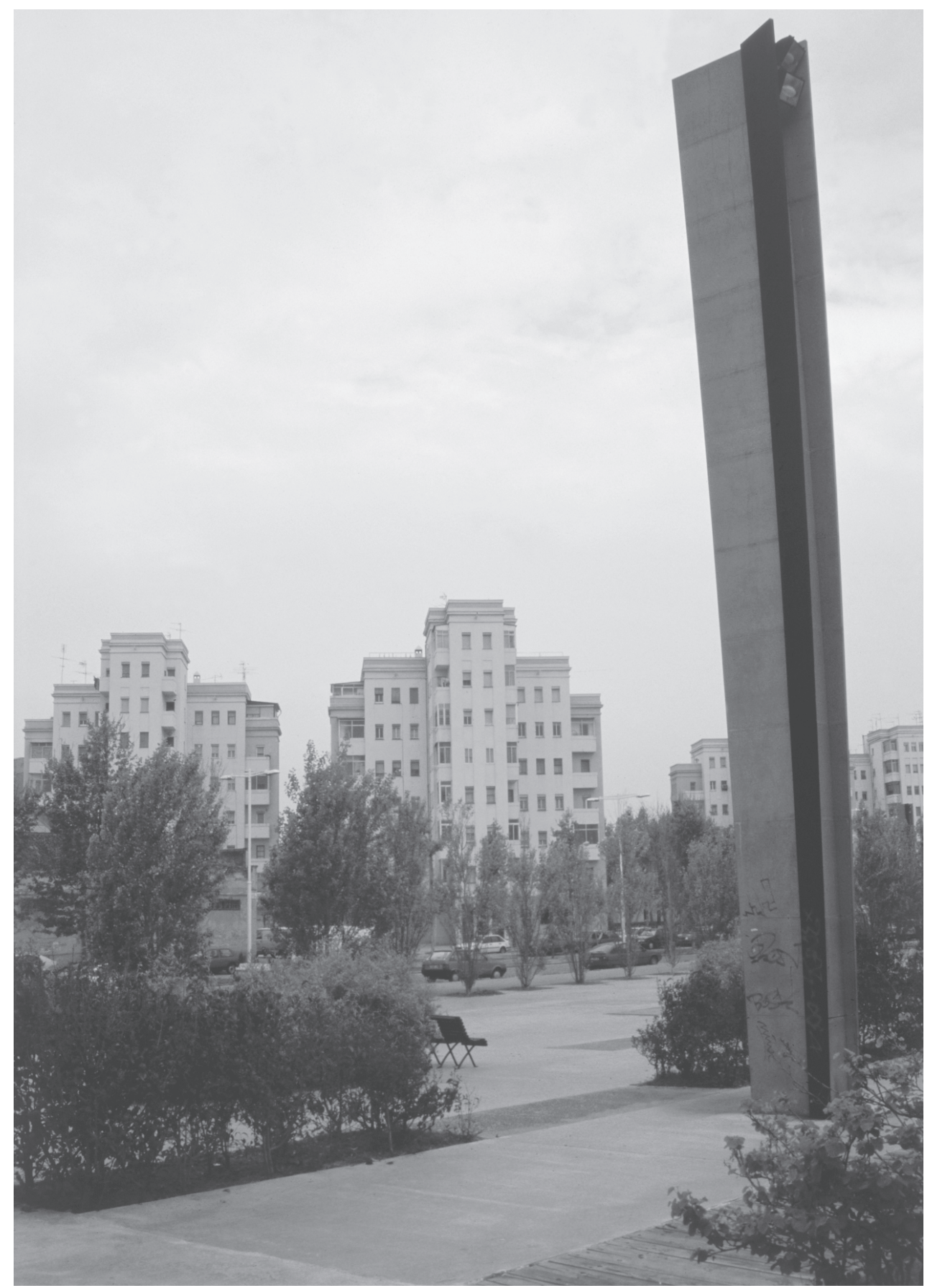

Foto de Vítor Matias Ferreira 


\section{La Dispersión de Población y Actividades sobre el Territorio}

La primera de las tendencias dominantes en la configuración del territorio metropolitano es hoy la dispersión de la urbanización sobre el espacio.

En efecto, la estructura territorial de la región metropolitana, tal como la conocemos hoy, es fruto de un largo proceso de concentración de población y actividades en el espacio catalán. Proceso que se remonta, por lo menos al siglo XVIII y a través del cual - a lo largo de la revolución agrícola y comercial del setecientos, de la industrialización del ochocientos y de la modernización de los tres primeros cuartos del novecientos - la población, acudiendo de allende de Cataluña y aún de otras regiones de España tiende a concentrarse en el espacio litoral y, especialmente, en el llano de Barcelona.

Fruto de este proceso de concentración son tanto las destacadas desigualdades en la distribución del poblamiento a las que nos referíamos más arriba como una de las características más notables de la ciudad de Barcelona: su elevadísima densidad (15 000 habitantes por $\mathrm{Km}^{2}$ ), a la cual es difícil encontrar parangón en otras ciudades europeas.

Pues bien, este proceso de concentración alcanzó su cenit en 1981 cuándo Barcelona llegó a su máximo poblacional histórico (1 752627 habitantes). Y desde entonces ha conocido una cierta reversión, una oleada descentralizadora y dispersiva que, de manera sorda e irregular, pero continua ha ido afectando el conjunto del territorio metropolitano. Así, en los últimos veinte años la ciudad de Barcelona ha perdido cerca de 250000 habitantes y ha pasado de contener el $40 \%$ de la población metropolitana al 35\%. Mientras, la primera corona metropolitana se mantiene estable desde el punto de vista demográfico (tanto en términos absolutos como relativos) y la segunda corona metropolitana crece de forma acelerada.

Nos encontramos pues ante un verdadero fenómeno de descentralización, con pérdidas netas de población del centro metropolitano que afectan no sólo Barcelona sino también los municipios que a ella se encuentran conurbados (como l'Hospitalet, Badalona o Santa Coloma, to- dos y cada uno de los cuales pirden población). Un fenómeno que, por otra parte, no es en modo alguno original, puesto que, como sabemos, afecta buena parte de las áreas metropolitanas españolas (así, de las siete mayores grandes ciudades españolas - Madrid, Barcelona, Valencia, Bilbao, Sevilla, Málaga y Zaragoza - las cuatro primeras conocen pérdidas netas de población en su municipio central en el último periodo intercensal).

El proceso descentralizador es el resultado de importantes migraciones residenciales intrametropolitanas. El análisis detallado de las mismas nos permite extraer dos consecuencias:

a) La configuración del espacio metropolitano ha pasado de estar estrechamente condicionada por migraciones inter-regionales asociadas al mercado de trabajo (en la fase previa a 1975) a depender sobretodo de migraciones intra-metropolitanas asociadas al mercado de la vivienda.

b) El fenómeno ante el que nos encontramos no es simplemente el de un desplazamiento desde el centro hacia la periferia metropolitana sino de un verdadero proceso de dispersión de población y actividades sobre el territorio, a través del cual la práctica totalidad de los núcleos con mayor población y densidad (independientemente de su localización) tienden a ceder peso relativo y, en muchos casos, población en términos absolutos hacia otras localidades más dispersas y de menor densidad.

La dispersión demográfica se corresponde también con la creciente dispersión de las actividades económicas y los servicios sobre el territorio metropolitano. La economía de la ciudad ha conocido durante los últimos veinte años un ajuste estructural muy notable: el paso de una economía de base industrial a otra de base crecientemente terciaria, así como a una mayor flexibilización de los procesos productivos (que ha afectado tanto el tamaño medio de las empresas, como la organización de los procesos productivos y las relaciones laborales). Pues bien, este doble proceso de ajuste se ha visto acompañado, desde el punto de vista territorial, por una notable tendencia hacia la descentralización de la ocupación sobre el espacio. 
En efecto, los datos disponibles muestran que Barcelona tiene todavía un peso muy notable sobre el total de la ocupación (en 1996 retenía todavía 659786 de los 1525090 lugares de trabajo existentes en la región metropolitana), pero pierde posiciones rápidamente: entre 1975 y 1996, la ciudad pasó de albergar el 56,2\% de los lugares de trabajo de la rmb al 43,3\% (mientras la primera corona se mantenía estable en términos relativos $23,7 \%$ en 1975 y 23,5\% en 1996 - y la segunda crecía de manera acentuada - del 20,1\% al $33,2 \%$. Los últimos datos de la Encuesta Metropolitana para el año 2000 sugieren que la tendencia habría continuado en los últimos cinco años, de forma que el peso de Barcelona superaría hoy apenas el $40 \%$ mientras el de la segunda corona alcanzaría ya más del $35 \%$.

\section{La Extensión del Área Urbana}

La segunda dinámica que caracteriza hoy la evolución de la metrópolis barcelonesa es su expansión sobre el territorio.

En efecto, al tiempo que su población y sus actividades se dispersan sobre el espacio, la región metropolitana se expande más y más, para integrar en su red de relaciones funcionales cotidianas un espacio cada vez más vasto.

Así, si tratamos de definir el ámbito barcelonés a partir de los criterios convencionales de las SMSA observamos como el territorio que podemos considerar metropolitano ha pasado de:

- 62 municipios y $1010 \mathrm{Km}^{2}$ en 1981, a

- 146 municipios en 1991, y a

- 216 municipios y $4597 \mathrm{Km}^{2}$ en 1996.

De esta manera el ámbito metropolitano estricto llega incluso a superar su delimitación administrativa a efectos de planeamiento (162 municipios) y se adentra decididamente hacia el interior de Cataluña y las provincias de Girona y Tarragona. En un futuro inmediato deberá seguirse atentamente el efecto que sobre esta dinámica expansiva tendrá la entrada en servicio (antes del año 2004) del tren de alta velocidad entre Barcelona, Girona, Lleida y Tarragona.

\section{La Especialización Funcional y Social}

Finalmente, las tendencias de dispersión y expansión de las dinámicas metropolitanas se ven acompañadas todavía de un tercer fenómeno: la especialización creciente de cada una de sus partes - de cada municipio, de cada barrio - en función del conjunto metropolitano.

Así, si bien es cierto que la dispersión de población, ocupación y servicios sobre el espacio metropolitano ha tendido a reducir las diferencias entre el centro y las coronas metropolitanas (incluso en términos de renta, como veremos más adelante), la creciente integración ha conllevado la especialización en términos funcionales de cada municipio. De este modo, residencia, comercio, industria y actividades de ocio y tiempo libre tienden a separarse cada vez más en el espacio metropolitano.

Esta especialización, se ve acompañada asimismo de una mayor segregación en términos sociales. Nos referíamos hace un momento a las migraciones metropolitanas, migraciones que como decíamos, tienen una relación directa con el mercado de la vivienda. Es decir, los flujos dominantes de movilidad residencial son inducidos y regulados, en muy buena medida, por las capacidad de los individuos y las familias de litigar en el mercado del suelo y vivienda. Se trata de un mercado que presenta rigideces muy importantes:

a) Según datos de la Encuesta Metropolitana 2000, el 84,6\% de la población metropolitana reside en viviendas de propiedad, lo cual deja el mercado de alquiler en una posición muy secundaria.

b) Por otra parte, la presencia y producción de vivienda de protección oficial es muy pequeña en comparación con lo que acontece en otros países europeos. Así, el año 2000 sólo 1 de cada 25 unidades de vivienda producidas en Cataluña gozaba de alguna forma de protección.

El esfuerzo económico necesario para la adquisición de vivienda es muy elevado (hasta 7,8 veces la renta familiar disponible anual para el caso de la vivienda nueva en Barcelona) y ha aumentado de forma notable en los últimos años pese a la 
reducción de los tipos de interés. En este contexto, el diferencial de precios entre la ciudad central y las coronas metropolitanas (los precios son en la ciudad, en términos 1,4 veces más elevados que en la primera corona y 1,8 más elevados que en la segunda) actúa como un acicate notable para la selección y salida de aquellos que, pudiendo adquirir vivienda en las coronas metropolitanas no alcanzan a hacerlo en la ciudad central (es decir, sectores de población mayoritariamente jóvenes y con niveles de formación e ingresos superiores a la media). Las diferencias de precios en el interior de cada una de las coronas actúan de la misma forma en la regulación de los movimientos de población.

Vemos pues que, en un contexto de rigidez del mercado, de precios elevados y de escasez de políticas públicas el mercado de la vivienda actúa como un poderoso filtro que condiciona estrechamente los procesos de segregación urbana.

\section{Los Beneficios de la Metropolitanización}

Vemos pues que en Barcelona, como en tantas otras metrópolis europeas, las transformaciones económicas y sociales de hoy comportan procesos de metropolitanización que están alterando radicalmente tanto la forma, como la función y la cohesión de los espacios urbanos.

Hay que decir, enseguida, que esta transformación comporta algunas consecuencias positivas muy notables, entre las que conviene destacar:

a) El incremento de la renta media. El proceso de metropolitanización (con la unificación del mercado laboral, la transformación de la base productiva y la aparición de nuevos sectores) ha sido, a un tiempo, causa y consecuencia del crecimiento económico experimentado en Cataluña y España en los últimos lustros. Crecimiento notablemente más elevado, en términos medios, al del resto de la Unió Europea, la cual cosa ha permitido que la renta media de Cataluña, que en año 1986 era todavía el 86\% de la Unió la haya igualado en el 2000.

b) El salto de escala metropolitano. Por otra parte, el paso de la ciudad desde los 2,5 a los 4,3 millones de habitantes efectivamente integrados en el mercado laboral y de consumo metropolitano ha dado a Barcelona una masa crítica que la pone en condiciones mucho mejores para atraer inversiones privadas, servicios, sedes de empresas e inversiones en obras públicos. Así, Barcelona constituye la sexta región metropolitana de Europa, superada sólo por Londres, París, el Ruhr, el Randstad y Madrid.

c) La igualación relativa de los niveles de renta. Se ha producido, asimismo, una cierta convergencia entre los niveles de renta de la ciudad y las coronas metropolitanas. Así, al desplazarse hacia las coronas población, actividades y servicios, éstas, que gozaban de niveles de renta media muy inferiores a los de la ciudad central han ido acercándose a ella. De esta manera, si la primera corona tenia en 1989 una renta media equivalente al $77,1 \%$ de la ciudad central alcanzaba ya el $85,3 \%$ en 1999. Y en el caso de la segunda corona se pasaba, en el mismo periodo del 79,8\% al 96,8\% de la media de Barcelona.

\section{Los Retos de la Transformación Urbana}

Ahora bien, es innegable que estas ventajas se han visto acompañadas de importantes problemas. Así, la ciudad, y el área metropolitana en su conjunto deben hacer frente hoy a notables desafíos de orden ambiental, funcional y social.

\section{La Problemática Ambiental}

En el orden ambiental, el problema sin duda más destacado es el consumo de suelo. Este ha conocido un aumento exponencial que ha llevado a transitar de la situación de 1882, cuando sólo $1763 \mathrm{Ha}$ de las 323000 que integran la rmb estaban ocupadas por la urbanización, a las 21482 de 1972 y las 45036 de 1992. En la actualidad el ritmo de transformación es cercano a $1000 \mathrm{Ha}$ al año, es decir cerca de $3 \mathrm{Ha}$ al dia. El resultado, como puede verse, es muy llamativo, e implica:

- Una hipoteca para los crecimientos y para las necesidad de interés público futuras. 
- El aislamiento y sacrificio de espacios de especial interés natural.

- El sacrificio de suelos de notable valor agrícola.

- La pérdida de valores paisajísticos.

- La impermeabilización y la artificialización de gran cantidad de suelo con el consiguiente incremento de los riesgos naturales.

Además el crecimiento disperso de la urbanización se traduce en un aumento de los consumos energéticos, en mayores dificultades para la recogida y el tratamiento de residuos, así como en un mayor gasto de agua por habitante y problemas para el saneamiento. Finalmente, la dispersión tiene también efectos muy notables sobre la movilidad.

\section{La Eficiencia Funcional}

En efecto, la expansión del ámbito metropolitano y la dispersión urbanización han comportado una explosión de las necesidades de movilidad de los ciudadanos y las empresas. En esta área urbana siempre más amplia, en la que cada lugar se especializa en función del conjunto, los ciudadanos realizan sobre un espacio mucho más vasto las funciones - residir, trabajar, comprar, disfrutar del tiempo libre - que antes satisfacían en un ámbito mucho más restringido.

Esto es bien visible en la evolución de la capacidad de autocontención de los municipios (es decir, la capacidad de cada uno de ellos de retener en su interior la movilidad que genera). Así en la provincia de Barcelona, los municipios que contenían menos del $50 \%$ de su movilidad laboral han pasado de:

- 102 de los 310 municipios en 1986, a

- 151 de los 310 municipios en 1991, a

- 208 de los 310 municipios en 1996.

De esta forma, en 4 de cada 5 municipios de la rmb por lo menos la mitad de los que trabajan lo hacen fuera del propio municipio y las tasa media de autocontención cayó del $67,6 \%$ al $55 \%$ entre 1986 y 1996. Los datos, muy recientes de la Encuesta Metropolitana para el año 2000 apuntan a una nueva caída de 7 puntos percentuales entre 1995 y el 2000.

Este incremento de la movilidad ha comportado un incremento extraordinario de la demanda de infraestructuras viarias y de transporte público. Sin embargo, al ser la inversión en este último campo muy inferior a la que se ha producido en la red viaria el reparto modal ha sufrido una modificación radical a favor de los desplazamientos en medios mecanizados privados.

Tabla 1 - Región Metropolitana de Barcelona. Reparto modal de los desplazamientos 1990-2000

\begin{tabular}{|l|c|c|}
\hline Tipo de desplazamiento & $\mathbf{1 9 9 0}$ & $\mathbf{2 0 0 0}$ \\
\hline Desplazamientos a pie & 26,9 & 21,1 \\
\hline Desplazamientos transporte público & 25,3 & 20,6 \\
\hline Desplazamientos transporte privado & 42,6 & 53,5 \\
\hline Otros desplazamientos & 5,2 & 4,8 \\
\hline Total & 100,0 & 100,0 \\
\hline
\end{tabular}

Fuente: Encuesta Metropolitana de Barcelona, 2000. 
Sólo en la ciudad de Barcelona y en los trayectos que a ella se encuentran asociados, el transporte público conserva todavía una cierta pujanza y representa algo más de un tercio de los desplazamientos.

Esta situación es, sin duda, el resultado de una política de inversiones (públicas y privadas) que ha primado en gran medida, la inversión en la red viaria en detrimento del transporte público. Pero es también consecuencia de la forma como se ha producido la ocupación del suelo en los últimos años. Así, según los datos de la agencia Barcelona Regional sólo el $2 \%$ de la superficie de la región metropolitana tiene una accesibilidad media al resto del territorio metropolitano igual o inferior a los 60 minutos. Pues bien, la dispersión de los últimos años ha llevado más población y actividades a situarse fuera de estas áreas de alta accesibilidad. Para entendernos:

a) Mientras el $44 \%$ del suelo residencial compacto está dentro del área de influencia directa (500 metros) de una estación.

b) Sólo el 6\% del suelo residencial disperso o el $11 \%$ del industrial gozan de esta condición.

Son pues los nuevos patrones en la ocupación del suelo, unidos a la escasez de inversión en transporte público, lo que está comportando problemas de eficiencia en las redes metropolitanas de movilidad. Problemas que se expresan en:

- Congestión de la red viaria y obsolescencia reiterada de las inversiones en este campo.

- Aumento del tiempo social dedicado a desplazamientos (aumento que afecta con particular intensidad los grupos sociales con menor capacidad para escoger lugar de residencia, y, en cada hogar, a las mujeres, los jóvenes y los ancianos).

- Aumento de la contaminación y la siniestralidad.

- Incremento del consumo de energía por ocupado y por lugar de trabajo.

\section{La Segregación Social}

Se ha explicado más arriba como el mercado de la vivienda actúa como un incentivo poderoso para la separación de los grupos sobre el territorio y, en particular, para la salida de grupos medios de la ciudad central hacia las coronas metropolitanas. Éstos fenómenos podrían hacer temer que se produjera una dualización de la ciudad, en el sentido que ésta pudiera devenir un lugar sólo para los muy ricos o para los muy pobres.

La evidencia estadística viene, afortunadamente, a desmentir esta hipótesis:

a) Como ya se ha explicado más arriba, las diferencias entre la ciudad central y las coronas metropolitanas, en términos de rentas medias, han tendido a disminuir en los últimos 15 años.

b) Pero, además, en la ciudad central, la distancia entre el primer y el último decil de la población en términos de renta media se ha reducido de 15 a 1 en 1985 a 10,5 a 1 en el año 2000.

c) Asimismo, la distribución total de la renta tanto en la ciudad central como en las coronas metropolitanas, ha tendido a hacerse más equitativa (como muestran los análisis basados en los cálculos de los índices de Gini).

Esta evolución positiva de las diferencias de renta ha de ser atribuida, en buena parte, a la introducción en España de los mecanismo básicos del Estado del bienestar (pensiones no contributivas, cobertura de desempleo, universalización de la sanidad) y de las políticas públicas a las que más adelante no referiremos.

Ahora bien, estos avances no niegan en modo alguno ni la persistencia de importantes desigualdades (el primer quintil de la población percibe el $40 \%$ de los ingresos totales, mientras el último recibe sólo el $7,3 \%$ ), ni la pervivencia y aún la agravación de situaciones muy problemáticas para algunos colectivos y territorios. Entre estos destacan, por la gravedad de los problemas que los afectan, un conjunto de barrios metropolitanos que representan el $6 \%$ de la superficie residencial, 180000 viviendas y cerca de 400000 habitantes, que según la administración deberían ser objeto de intervenciones de "rehabilitación integral". Se trata, sobretodo, de áreas centrales envejecidas y de polígonos de vivienda construidos en los años 60 y 70, así como algunas áreas de urbanización marginal. És aquí donde tienden a concentrarse hoy los sectores con menor poder adquisitivo y la nueva inmigración extracomunitaria. 


\section{Las Estrategias de Futuro}

Hemos tratado de mostrar como la región metropolitana de Barcelona se encuentra hoy sujeta a un acelerado proceso de cambio. La ciudad, que se ha caracterizado tradicionalmente por su compacidad física, por la complejidad de usos en su interior y por la convivencia de grupos sociales muy diversos, está pasando a ser más dispersa, más especializada y más segregada.

Como se ha mostrado, ésta trasformación comporta oportunidades y retos para el futuro de la ciudad central y su área. Estos son particularmente importantes para la ciudad central que ve como, dejada a ella misma (es decir, en caso de no regularse de alguna forma los impulsos del mercado) podría convertirse en un espacio crecientemente ineficiente desde el punto de vista funcional, insostenible desde el punto de vista ambiental e insolidario desde el punto social.

Las políticas urbanas que se han tratado de aplicar desde el Ayuntamiento de la ciudad en las últimas dos décadas han tenido, en buena medida, como objetivo esencial hacer frente a estos retos. Así, de forma implícita o explícita, se ha tratado de defender un modelo de ciudad razonablemente compacta, compleja en cuanto a sus usos e integrada en cuanto a su población. Entendiendo que compacidad, complejidad y cohesión como requisitos esenciales para la eficiencia, la sostenibilidad y la equidad: el sprawl, la ciudad dispersa no es ciudad, es a lo sumo urbanización, en la que aquellos elementos que hacen atractiva y deseable la vida urbana contemporánea - la innovación, la riqueza de usos, la capacidad de compensar las desigualdades - brillan por su ausencia.

En esta defensa de la ciudad, la renovación urbana ha jugado un papel esencial. Y ha conseguido éxitos muy notables, como la rehabilitación de Ciutat Vella, la intervención en el Poblenou o la política de espacios públicos en todos los distritos, fueran estos céntricos o periféricos. Ante el futuro, las nuevas políticas de renovación y defensa de la ciudad central deberán capaces de dotarse de visión, organización y instrumentos para resolver cuatro cuestiones básicas:

a) La integración en la red. En un mundo de flujos, la ciudad debe ser un lugar lo sufuciente mente atractivo para ejercer la función de bisagra entre las lógicas de orden global y las de orden local. Esto implica asegurar su accesibilidad ex- terior, su capacidad de innovación, así como las diversidad y convivencia en su interior de personas con orígenes y expectativas muy variadas. Accesibilidad exterior, capacidad de innovación y diversidad interior son las claves para asentar firmemente la ciudad como nodos de la red.

b) La articulación de la metrópolis. La ciudad compacta - las ciudad intensa como alguna vez la hemos denominado - está en mejor posición que otras para hacer frente a estos retos. Pero nos equivocaríamos si al afirmar la ciudad compacta quisiéramos negar la metropolitanización o basar su desarrollo en la potenciación de un único centro urbano. Si así lo hiciéramos, la presión sobre la ciudad central sería tan intensa que se traduciría - a través de los precios - en nuevos procesos de segregación y exclusión. Lo que debemos perseguir es una metrópolis articulada por un conjunto de ciudades integradas en una red. Una metrópolis polinodal. Como una ciudad formada por ciudades: una ciudad de ciudades.

c) La centralidad de la política. Para avanzar hacia esta configuración urbana hacen falta valores y proyectos colectivos capaces de condicionar y de contradecir, en los casos que haga falta, los impulsos del mercado. Hay pues que reivindicar la centralidad de la política en la construcción del espacio urbano. Navegar a favor de la corriente no es gobernar. Gobernar es conseguir que ocurra aquello que sin la decisión y la voluntad colectiva no ocurriría.

Esto no significa, sin embargo, que debamos ser esclavos de los viejos planes, de los esquemas fijos. Toda política que sea fija quedará pronto obsoleta. Así, la defensa e impulso de la renovación urbana debe pasar por un

- Urbanismo flexible más preocupado por el proceso de construcción de la ciudad que por la persecución de una imagen-objetivo final.

- Urbanismo de gestión y estratégico, capaz de considerar, junto al contenido físico, las opciones decisivas para la transformación económica y social.

- Urbanismo participativo, con interacción electrónica, ejercicios de simulación y consejos ciudadanos.

- Urbanismo, en fin, que inscriba la sostenibilidad, la eficiencia y la equidad como objetivos esenciales.

La ciudad de calidad no es aquella que pretende cancelar sus conflictos - y por esta quiméri- 
ca vía alcanzar la calidad - sino aquella que es capaz de gestionar estas contradicciones a través de procesos democráticos y proyectos colectivos.

d) La innovación con raíces. El tipo de renovación urbana que se deriva de estos postulados debe ser capaz de combinar transformación con memoria, creación con herencia, progreso con equidad. Y ello, en la ciudad central, requiere una política urbana que:

- Establezca proyectos culturales como motor y como irradiación

- Defienda y refuerce el espacio público

- Busque la conexión entre el sustrato histórico y las actividades de nuevo tipo

- Evite los desplazamientos salvajes de población y la formación de ghettos

- Prime la rehabilitación ante la demolición

- Vincule los usos del suelo a la accesibilidad

- Regule y domestique el tráfico

En dos palabras: el futuro de la ciudad central depende hoy, por una parte, de su capacidad para renovarse a través de los valores de convivencia e innovación, y, por otra, de su capacidad de liderar un territorio vertebrado y de alta calidad ambiental. Pues bien, esta capacidad esta relacionada de forma directa con la habilidad de la ciudad para crear, impulsar y mantener actividades en sectores innovadores y emergentes.

\section{El Papel de los Sectores Emergentes: tres ejemplos}

Veamos, pues, para finalizar tres ejemplos de la forma como las políticas de renovación urbana aplicadas en la ciudad de Barcelona están tratando de aplicar, en la práctica estos valores. Se trataran tres ejemplos, que, por su envergadura, tienen implicaciones en todos los aspectos de la vida ciudadana. Sin embargo, por sus características, cada uno de ellos tiene, respectivamente, un impacto especial sobre la compacidad, la complejidad de usos y la cohesión social de la ciudad.

\section{La Actividad Turística, Cultural y los Nuevos Polos para el Tiempo Libre}

La actividad turística es un sector en clara expansión en la ciudad de Barcelona. Si bien es cierto que Cataluña, en particular las áreas litorales de la Costa Brava y la Costa Dorada, constituye uno de los principales destinos turísticos del Mediterráneo, la ciudad había quedado relativamente al margen de los grandes flujos turísticos. Así, sus visitantes lo eran en la mayoría de los casos por razones de negocios, y en particular por la actividad ferial que cuenta con una larga tradición en la ciudad.

La situación ha conocido un cambio radical desde la celebración de los Juegos Olímpicos de 1992. Estos tuvieron, desde el punto de vista del impulso de la actividad turística una doble virtud:

a) Por una parte, constituyeron un colosal ejercicio de promoción para la ciudad, que permitió proyectar una imagen basada, en muy buena medida en sus atractivos culturales y arquitectónicos.

b) Por otra parte, la atracción de inversión pública y privada hacia la ciudad - que tuvo efectos muy notables en el campo de las infraestructuras y los equipamientos - incluyo también la realización de un "Plan de hoteles". Este permitió pasar de 118 establecimientos y 18569 plazas en 1990 a 148 establecimientos y 25055 plazas en 1992 . Desde entonces la oferta no ha dejado de crecer y permitía alcanzar el año 2000 las cifras de 187 establecimientos hoteleros y 31338 plazas. Hasta el año 2004 se prevé la incorporación de 7500 nuevas plazas.

Paralelamente, la demanda ha conocido una verdadera explosión, que la ha llevado a pasar de 3,8 millones de pernoctaciones en 1990 a más de 7,7 millones en el 2000, doblándose, pues en una década. Sólo el $29 \%$ de los visitantes son españoles, y entre el resto, destacan, por países, los que proceden de los Estados Unidos (13,5\%), Gran Bretaña $(11,2 \%)$, Italia $(6,3 \%)$ o Alemania $(5,8)$.

Otro elemento que ha conocido un cambio muy notable son los motivos de desplazamiento. Mientras a inicios de la década de los noventa, las visitas por negocios superaban ampliamente el $50 \%$ del total, en el año 2000 constituían sólo el 36,7\%, mientras que el motivo turístico alcanzaba ya el 43,9\%. Se trata, además, de un turismo con un alto interés cultural que, según las encuestas realizadas, valora como los tres principales atractivos de la ciudad su oferta arquitectónica, su oferta cultural y el entretenimiento. 
Este incremento de la demanda exterior en el sector de la oferta cultural se complementa con un aumento muy notable de la demanda interior. Una parte considerable de la población de la rmb considera que su disponibilidad de tiempo libre es muy alta $(29,1 \%)$ o bastante alta $(34,5 \%$, aunque, dicho sea de paso, este tiempo se obtiene a menudo robando horas al sueño: en la ciudad de Barcelona, en el año 2000, el 60,2\% de la población afirma que se va a dormir entre medianoche y la una de la madrugada, mientras que el 71,6\% afirma que se levanta entre las cinco y las ocho de la mañana). De entre la población adulta las actividades de tiempo libre que se practican "a menudo" son: ir a la playa $(42,2 \%)$, al cine $(19,9 \%)$, a restaurantes $(16 \%)$ y a museos y exposiciones $(8,6 \%)$.

La política urbanística ha tratado de asociar la emergencia de esta nueva demanda con la creación de nuevos polos para actividades de tiempo libre en el interior mismo de la ciudad. Polos que han sido utilizados además como instrumento de rehabilitación de unas áreas y dinamización de otras. Se ha hecho pues, en conjunto, una apuesta por el turismo y el ocio cultural y urbano que contrasta con la dispersión de la urbanización, el consumo de suelo y la banalización del paisaje que ha comportado el desarrollo turístico del litoral.

Los principales de estos nuevos polos de consumo cultural y tiempo libre son los siguientes:

a) El eje cultural de Ciutat Vella. Eje que cruza el Raval, en el centro del casco histórico desde el puerto hasta la Plaza de la Universidad. Aquí han sido colocados, en el corazón de un barrio con profundos problemas de envejecimiento y degradación algunos de los principales equipamientos culturales de la ciudad y de Cataluña: el Museo de Arte Contemporánea de Barcelona, el Centre de Cultura Contemporánea de Barcelona, el Centre de Recursos Culturals, la sede de uno de los principales grupos editoriales catalanes, la renovada Biblioteca Nacional y la sede del Consejo Superior de Investigaciones Científicas.

b) El eje litoral. De la Barceloneta hasta el Poblenou y la Marbella se han recuperada cerca

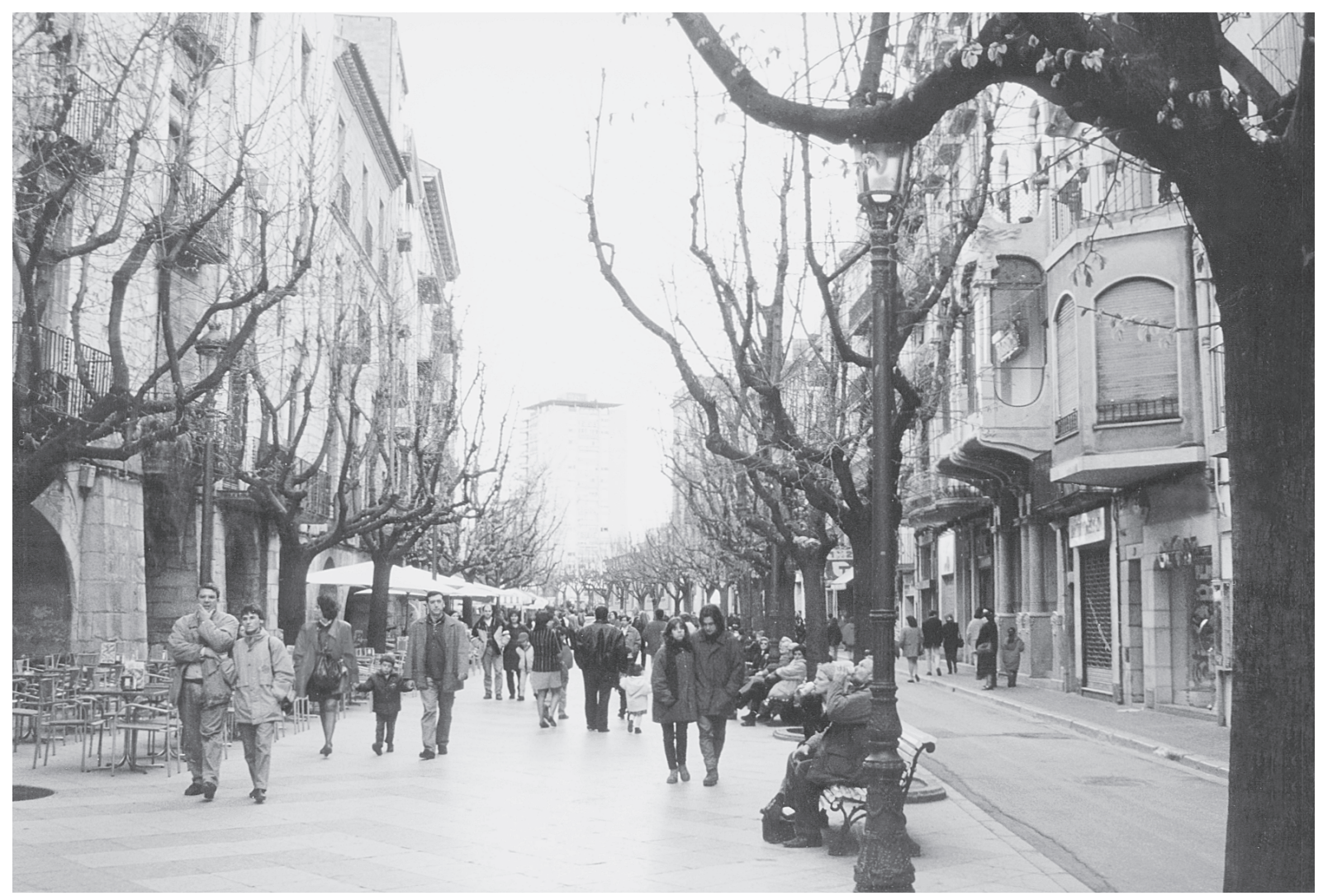

Foto de Vítor Matias Ferreira 
de cinco kilómetros de frente marítimo urbano, que han sido adecuados para el baño y el ocio, con la construcción de un paseo marítimo, el Puerto Olímpico, y la instalación de numerosos restaurantes y bares. Esta apertura de la ciudad al mar, que ha de ser considerada, sin duda uno de los principales legados del urbanismo olímpico, ha permitido modificar radicalmente la oferta turística de la ciudad (que puede ahora ofrecer, tambien, producto de sol y playa) y los hábitos de tiempo libre de los barceloneses (el frente marítimo se ha convertido en la playa preferida para muchos de los habitantes de la ciudad: el $27,2 \%$ de los que van a la playa afirman que su destino principal es este).

c) El Port Vell. La parte interior del puerto de Barcelona, contigua al centro histórico de la ciudad, ha sido asimismo recuperada para el uso ciudadano, con la remoción de las barreras que antaño impedían el acceso a la primera línea de mar y la construcción de pasarelas que permiten el acceso a los antiguos muelles. Aquí se ha instalado el polo del Maremagnum, íntegramente dedicado al tiempo libre, donde se ha instalado un centro comercial (con tiendas, bares y restaurantes), el nuevo acuario, un complejo de cines y una sala IMAX. El volumen construido en esta área es muy considerable, lo cual ha levantado críticas respecto a la oportunidad de levantar un complejo tan singular en primera línea de mar, en un lugar que presenta además problemas de accesibilidad muy notables.

d) Los nuevos centros comerciales urbanos. Finalmente, la ciudad ha hecho una apuesta decidida para evitar el desplazamiento del comercio hacia el entorno metropolitano. Así, se han reequipado e integrado los espacios comerciales tradicionales, dándoles una imagen de marca y una promoción homogénea (Barnacentre, eix comercial de Sant Andreu, Creu Coberta). Y por otra parte, se ha tratado de evitar la instalación exclusiva de los grandes centros comerciales en las coronas metropolitanas, sobre las grandes arterias de tráfico. Así, utilizando vacíos urbanos (fruto del desplazamiento de la industria o de infraestructuras) para anclar algunos de estos centros en el interior mismo de la ciudad central (Illa Diagonal, la Maquinista, Glóries, Heron, Diagonal Mar), tratando que su instalación sea no un factor de desertización de la ciudad sino de irradiación de actividades en su entorno.

\section{Las Nuevas Actividades Productivas y el Sector22@}

Otra intervención que traduce una voluntad clara de defender los valores de la compacidad y complejidad en el desarrollo urbano es la operación $22 @$ en el área del Poblenou.

En esta área, que, como se ha dicho, ha sido tradicionalmente el centro industrial a levante de la ciudad (el otro se encuentra a poniente, hacia el Delta del Llobregat), se ha propiciado una modificación del Plan General Metropolitano para permitir la instalación de nuevas actividades y la mezcla de usos (la modificación fue aprobada de forma definitiva el 27 de Julio del 2000). Así, la vieja calificación de suelo industrial ha sido transformada en una nueva clave, denominada 22@, que, de forma innovadora, pretende atraer actividades vinculadas a sectores emergentes de la producción y la creación (producción de software, telecomunicaciones, multimedia, prensa, empresas de procesos de datos y comercio electrónico, actividades artísticas y centros de investigación y enseñanza).

Estos nuevos usos deberían generar, en los próximos años, alrededor de 100000 nuevos lugares de trabajo, y se podrán combinar con la existencia de usos residenciales en la misma área. Se trata pues de un intento de atraer actividades productivas emergentes, a caballo entre la industria y los servicios, hacia el centro de la ciudad. Atraerlas e instalarlas en un área en la que, rompiendo los rígidos esquemas del zonning se encuentren mezcladas con residencia y centros de producción artística, docencia e investigación.

Las magnitudes de la operación son las siguientes:

- El área afectada por la calificación 22@ es de 198,3 Ha.

- Se prevé la transformación de 1159626 $\mathrm{m}^{2}$ de suelo industrial, con un potencial total de nuevo techo construido (equipamientos excluidos) de aproximadamente $3500000 \mathrm{~m}^{2}$.

- La oferta de techo construido para nueva actividad económica será de 3200000 m².

- La inversión necesaria en bienes de equipo necesaria para desarrollar las actividades productivas del nuevo sector se estima en 700000 millones de pesetas.

- Se reconocerán 4614 viviendas que actualmente existen sobre suelo industrial y se producirán una 3500 viviendas nuevas en régimen de protección oficial. 
- El aumento de zonas verdes será de unos $75000 \mathrm{~m}^{2}$ y el suelo destinado a nuevos equipamientos de $145000 \mathrm{~m}^{2}$.

- El potencial inmobiliario de la operación, en pesetas 2001, es de 1,3 billones de pesetas.

\section{El Futuro de la Ciudad y el Forum 2004}

Si las dos intervenciones anteriores tenían relación sobretodo, con la defensa de los valores de compacidad y complejidad, la última a la que nos queremos referir tiene, además, un marcado acento social, una voluntad explícita de incidir en la cohesión social de la ciudad. Se trata de la operación del Forum 2004 en el frente litoral del Besòs.

El área constituye un verdadero compendio de problemática urbana, en el que se unen la presencia de grandes infraestructuras energéticas y ambientales (una planta depuradora, cinco centrales térmicas, una planta de incineración de residuos), la desembocadura de un río - el Besòs con una elevadísima contaminación, grandes infraestructuras viarias (la Diagonal - inconclusa - el Cinturón del Litoral), polígonos de vivienda de masa de los años sesenta y setenta con una problemática social notable (la Mina, Sant Ramon de Penyafort, la Catalana), la existencia del límite administrativo entre Barcelona y el municipio vecino de Sant Adrià del Besòs.

La magnitud y la diversidad de los problemas planteados ha llevado al diseño de una operación muy compleja, que incluye la aplicación de diversos proyectos específicos y la organización de un gran evento internacional. En efecto, el recurso de la ciudad de Barcelona - privada de capitalidad de un Estado y postergada, a menudo, desde el punto de vista de las inversiones públicas - a la organización de un evento para impulsar la transformación urbana ha sido una constante en los últimos 150 de historia de la ciudad. Así se han organizado las Exposiciones Universales de 1888 y 1929, el Congreso Eucarístico de 1952 y los Juegos Olímpicos del 1992.

En este caso, se trata de un evento concebido desde la propia ciudad, que recibe el nombre del "Forum Universal de las Culturas, Barcelona 2004". El Forum quiere así convertirse en un "Nuevo tipo de evento global, organizado para promover el diálogo entre gentes de todas la culturas.
Se realizaran debates y discusiones de todas la cuestiones de interés común, así como un Festival Mundial de las Artes y Exposiciones temáticas sobre la diversidad humana y su historia. Será una gran fiesta, una expresión de la creatividad de todos los pueblos y un encuentro político de dimensiones globales con nuevos e imaginativas formas de participación, centrado en uno de los principales desafíos del siglo XXI: el diálogo de las culturas."

El evento, al que, como se puede ver, no se le puede negar la ambición, tendrá lugar durante el verano del año 2004 y ha recibido el reconocimiento y el apoyo de la UNESCO.

Desde el punto vista de la intervención urbanística el plan que afecta un conjunto de 170 Ha., prácticamente contiguas, al Distrito 22@al que nos referíamos más arriba e incluye las operaciones siguientes:

- La construcción de una plataforma, sobre terrenos ganados al mar, que constituirá el nuevo frente marítimo del área, con playas, paseo marítimo y parque urbano, y al que se trasladará, en parte, el zoo de la ciudad desde el parque de la Ciutadella.

- La construcción de un nuevo puerto deportivo junto a la desembocadura del Besòs, con capacidad, en principio para 2000 amarres.

- La construcción de los espacios del Forum, con un edificio central, una gran plaza y una explanada que cubrirá la depuradora y, en parte, el eje viario del Cinturón del Litoral.

- La remodelación del barrio de la Mina, y de los sectores de Llull-Taulat y la Catalana, para la cual se cuenta con la contribución del programa URBAN de la Unió Europea y se ha creado un consorcio interadministrativo específico. La reordenación de la infraestructuras de tratamiento de residuos y producción de energía.

- La dotación de grandes equipamientos metropolitanos, entre los que se cuentan el Palacio de Congresos de Cataluña y un nuevo campus universitario, dedicado, en parte a la investigación en ciencias bio-médicas.

El éxito de la operación presenta todavía notables incógnitas. Es, pero, un nuevo intento, con el que Barcelona trata de combinar innovación económica, renovación urbana y cohesión social. Es decir, de afrontar algunos de los principales retos del futuro de la ciudad. 